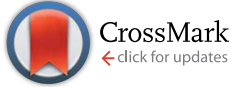

Cite this: RSC Adv., 2017, 7, 16650

Received 20th December 2016 Accepted 27th February 2017

DOI: $10.1039 / c 6 r a 28465 e$

rsc.li/rsc-advances

\section{Reduced graphene oxide (RGO) enriched polymer network for highly-enhanced electro-optic performance of a liquid crystalline blue phase $\uparrow$}

\author{
MinSu Kim, $₫ \S^{a b}$ R. K. Mishra, $\ddagger^{a}$ Ramesh Manda, ${ }^{a}$ G. Murali, ${ }^{a}$ Tae-Hyung Kim, ${ }^{c}$ \\ Myong-Hoon Lee, ${ }^{c}$ Minhee Yun, ${ }^{d}$ Sudarshan Kundu, ${ }^{e}$ Byoung-Suhk Kim ${ }^{f}$ \\ and Seung Hee Lee*a
}

\begin{abstract}
The excellent physical and chemical properties of graphene have been extensively studied for both scientific interest and novel applications in devices, such as organic photovoltaics, fuel cells, biosensors, and display devices. One of its excellent two-dimensional electric properties is its high electron transporting capability which gives enormous possibility for the performance enhancement of numerous electronic devices. Herein, we demonstrate the record-breaking electro-optic performance of a polymer-stabilized liquid crystalline blue phase using a reduced graphene oxide (RGO) enriched polymer network, which not only enhances the conductivity in the system but also provides counter-benzene-rings to liquid crystalline molecules for strong $\pi-\pi$ interaction. It reduces the operation voltage $(\sim 32 \%)$, response time $(\sim 51 \%)$ and hysteresis $(\sim 53 \%)$ compared to that of a conventional polymer-stabilized BPLC, and thereby possibly contributes to novel electro-optic device applications.
\end{abstract}

\section{Introduction}

Graphene, which is a monolayer of carbon atoms tightly packed into honeycomb lattices, is the first theoretical and experimental example of a truly two-dimensional (2D) nanomaterial. Substantial scientific studies are widely devoted to graphene due to its excellent chemical and physical characteristics, such as crystallographic quality, low resistivity, high chemical stability, good optical transmittance, ballistic electron transportation and excellent mechanical strength., ${ }^{1,2}$ Since the

\footnotetext{
${ }^{a}$ Applied Materials Institute for BIN Convergence, Department of BIN Convergence Technology, Department of Polymer-Nano Science and Technology, Chonbuk National University, Jeonju, Jeonbuk 561-756, Korea. E-mail: lsh1@chonbuk.ac.kr; Tel: $+82-63-270-2343$

${ }^{b}$ Liquid Crystal Institute, Kent State University, Kent, $\mathrm{OH} 44242$, USA

${ }^{c}$ Graduate School of Flexible \& Printable Electronics Engineering, Chonbuk National University, Jeonju, Jeonbuk 561-756, Korea

${ }^{d}$ Department of Electrical and Computer Engineering, Swanson School of Engineering, University of Pittsburgh, Pittsburgh, PA 15261, USA

${ }^{e}$ Department of BIN Convergence Technology, Chonbuk National University, Jeonju, Jeonbuk 561-756, Korea

${ }^{f}$ Department of Organic Materials \& Fiber Engineering, Department of BIN Convergence Technology, Chonbuk National University, Jeonju, Jeonbuk 561-756, Korea

$\dagger$ Electronic supplementary information (ESI) available: Polarizing optical micrographs and electro-optic characterization of various concentration of RGO. See DOI: 10.1039/c6ra28465e

\$ These authors contributed equally to this work.

$\S$ Current affiliation: Department of Physics and Astronomy, Johns Hopkins University, Baltimore, MD 21218, USA.
}

successful synthesis of graphene oxide ${ }^{3}$ and reduced graphene oxide (RGO), ${ }^{4}$ such excellent properties are promising to realize ideal optically transparent conductors and numerous vital applications in devices including organic photovoltaics, biosensors, supercapacitors, fuel cells, display devices and nano-hybrid reinforcement. ${ }^{5-18}$ In addition to the number of well-known applications, one promising approach for organic electronics is graphene-enriched polymer composites including nanostructured polymer matrices. ${ }^{19,20}$

Liquid crystals (LCs) are one of the most widely used electrooptic materials. The LC and polymer composite is well-known as LC droplets dispersed in a polymer matrix, or the so called polymer-dispersed liquid crystal (PDLC) for light shutters, smart windows, and transparent displays. ${ }^{21}$ In the last decade, the blue phase liquid crystal (BPLC) has been one of the fascinating candidates for next generation display applications because of its unique electro-optic, photonic properties and fast response time, which is enabled by polymer-stabilization..$^{22}$ The structural property of the BP is based on 3D structured fluids, which are in a thermodynamically stable state with a sharp energetic balance between elasticity and defects. ${ }^{2,24}$ At sufficiently high-chirality, the double-twist formation becomes even in a low-energy state and thereby, is stabilized by the $3 \mathrm{D}$ selfassembly of the double-twist cylinder (DTC) and disclinations. By photo-polymerization, a polymer network is generated in the disclinations by pre-mixed monomers, and it compensates the energy cost to stabilize the phase by more than $80 \mathrm{~K}^{25,26} \mathrm{Such}$ polymer-stabilized (PS) BP has been of substantial interest in condensed matter physics, nanotechnology, and fast-response 

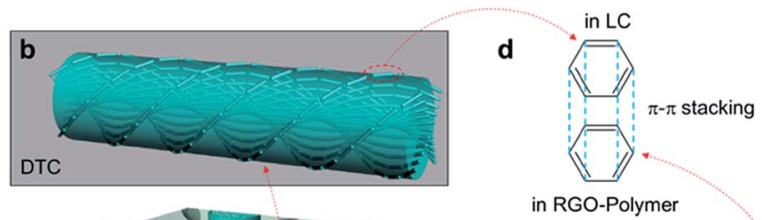

a
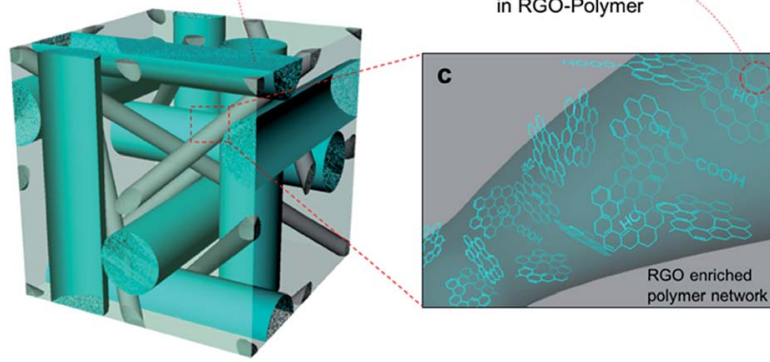

Fig. 1 Schematic of the RGO enriched PSBP. (a) PSBP lattice with RGO-polymer. (b) Double-twist cylinder. (c) RGO-polymer network. (d) $\pi-\pi$ interaction between LC and RGO-polymer.

light modulators including display device applications. However, there are critical shortcomings, such as highoperation voltage and electro-optic hysteresis, although its fast-response and optical isotropy can be huge advantages. ${ }^{22,25-32}$ In addition to the many efforts to reduce the operation voltage by adjusting electrode structures, ${ }^{27,28,30,31}$ recently, a report demonstrated that mixing small amount of a polyaniline functionalized graphene oxide (G-PANI) can effectively enhance the operation voltage of PSBP. ${ }^{19}$ In this way, additional processes of chemical treatments are required to produce GPANI.

Herein, we propose a novel RGO enriched PSBP using a polymerizable chiral agent. General steps are required to produce the RGO in this way, keeping the fabrication process relatively simpler than using G-PANI. First, the conductivity of the system can be enhanced by the RGO additive. Second, owing to the polymerizable chiral agent in the prepolymer mixture, a polymer network can be generated not only in disclination of BP but also inside the DTCs, giving rise to a relatively rigid polymer network. Third, it is known that ionic impurities can be trapped by the RGO-polymer composite, ${ }^{33,34}$ and thus the ionic effect would be suppressed. Finally, large numbers of counter benzene rings, against the benzene rings in LC, can be provided by RGO with respect to the polymer network intensifying $\pi-\pi$ interaction. Therefore, we expect that the anchoring strength between the LC and RGO-polymer composite would be greatly enhanced [Fig. 1].

\section{Results and discussion}

After photo-polymerization of the $\mathrm{BP}\left(72^{\circ} \mathrm{C}\right)$ on the PSBP-RGO0.05 sample, the BP existed over $\sim 11{ }^{\circ} \mathrm{C}\left(83-71{ }^{\circ} \mathrm{C}\right)$ [Fig. 2], whereas it was $\sim 2{ }^{\circ} \mathrm{C}$ before the polymer-stabilization. Although the stabilized temperature range is relatively narrower because the polymer network is formed not only in the disclinations but also inside the DTCs in the lattice by combination of RM-257 and the polymerizable chiral agent, the range could be extended by additional isometric reactive monomers, as

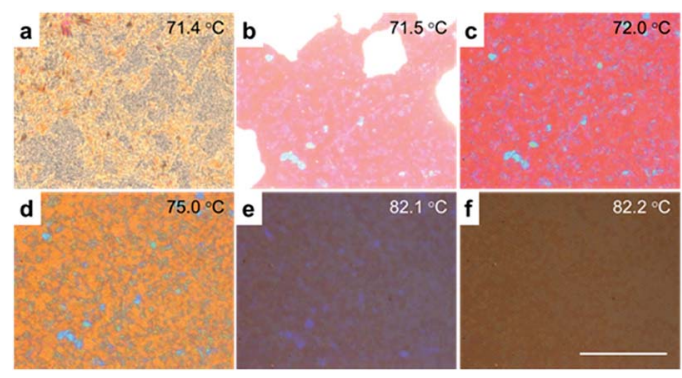

Fig. 2 POM images of the PSBP-RGO-0.05 cell at (a) $71.4^{\circ} \mathrm{C}$, (b) $71.5^{\circ} \mathrm{C}$, (c) $72.0^{\circ} \mathrm{C}$, (d) $75.0^{\circ} \mathrm{C}$, (e) $82.1^{\circ} \mathrm{C}$ and (f) $82.2^{\circ} \mathrm{C}$. Scale bar is 50 $\mu \mathrm{m}$.

reported in several previous studies. ${ }^{22,25}$ In order to verify the effect of the RGO-polymer composite, one can compare the relative values of the electro-optic properties with and without RGO, that is, between PSBP and PSBP-RGO. Thus, the static and dynamic electro-optic properties were measured by voltagedependent transmittance [Fig. 3(a)] and time-dependent transmittance [Fig. 3(b)].

Furthermore, the threshold, $V_{\text {th }}$, operation voltage, $V_{\text {op }}$, response time and hysteresis of all the samples are shown in Table 1 . As expected, ${ }^{\mathbf{1 9}}$ the operation voltage tends to be reduced as the concentration of RGO increases, and with $0.2 \mathrm{wt} \% \mathrm{RGO}$, the $V_{\text {th }}$ and $V_{\text {op }}$ are reduced by $45 \%$ and $31.7 \%$, respectively. In addition to the rise time, the decay time implies a pure interaction between the LC and RGO-polymer when removing the applied voltage. Although the measured decay time is relatively faster (less than sub-milliseconds) due to the measurement at
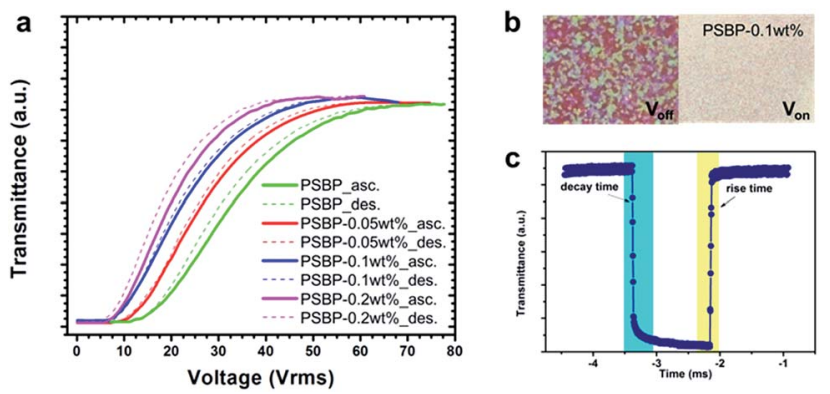

Fig. 3 Measured (a) V-T curves (solid: ascending; dotted: descending), (b) POM images in the $V_{\text {off }}$ and $V_{\text {on }}$ states at $V_{\text {op }}=48 V_{\text {rms }}$, and (c) the rise and decay times of the PSBP-RGO-0.1 cell at $74{ }^{\circ} \mathrm{C}$.

Table 1 Electro-optic performances measured at $74{ }^{\circ} \mathrm{C}$

\begin{tabular}{lllll}
\hline Electro-optics & $V_{\text {th }}\left[\mathrm{V}_{\mathrm{rms}}\right]$ & $V_{\mathrm{op}}\left[\mathrm{V}_{\mathrm{rms}}\right]$ & $\begin{array}{l}\text { Decay time } \\
{[\mu \mathrm{s}]}\end{array}$ & $\begin{array}{l}\text { Hysteresis } \\
{[\%]}\end{array}$ \\
\hline PSBP & 16 & 60 & 77 & 2.98 \\
PSBP-RGO-0.05 & $12(25 \%)^{a}$ & $53(12 \%)$ & $57(26 \%)$ & $1.41(53 \%)$ \\
PSBP-RGO-0.1 & $9.5(41 \%)$ & $48(20 \%)$ & $38(51 \%)$ & $1.53(49 \%)$ \\
PSBP-RGO-0.2 & $8.8(45 \%)$ & $41(32 \%)$ & $142(-84 \%)$ & $3.17(-6 \%)$
\end{tabular}

${ }^{a}$ The approximate degree of difference when RGO is mixed in PSBP in the parenthesis. 
high-temperature $\left(74^{\circ} \mathrm{C}\right)$, the comparison between PSBP and PSBP-RGO fairly demonstrates how RGO influences the electrooptics. The decay time of PSBP-RGO-0.05 is reduced by $\sim 26 \%$ compared to PSBP, and that of PSBP-RGO-0.1 is even further reduced by $\sim 51 \%$. The hysteresis is defined as $H=\left[\left(V_{\mathrm{asc}}-V_{\mathrm{des}}\right) /\right.$ $\left.V_{\mathrm{p}}\right] \times 100 \%$, where $V_{\text {asc }}$ and $V_{\text {des }}$ are half of the peak voltages $V_{\mathrm{p}}$ in ascending and descending, respectively. ${ }^{25}$ Again, comparing PSBP-RGO-0.05 and PSBP, the hysteresis drops by 53\% owing to the RGO-polymer composite.

Comparing Fig. 4(a) and (b), it is observed that the electrooptic properties rely on different regimes, that is, a linear dependence on the $V_{\text {th }}$ and $V_{\text {op }}$ and non-linear dependence for the decay time and hysteresis. This result is partially consistent with the previous report in ref. 19 which utilized G-PANI. In the previous study, $V_{\text {op }}(\sim 19 \%)$ was reduced by 1 wt $\%$ of G-PANI (but when the G-PANI concentration range was $0.05-0.5 \mathrm{wt} \%$, it increased), whereas it was $\sim 32 \%$ reduced by only $0.2 \mathrm{wt} \%$ RGO in our study. The decay time and hysteresis in that previous study become significant by increasing the G-PANI concentration higher than $0.5 \mathrm{wt} \%$ (but to similar degree when the G-PANI concentration was below $0.5 \mathrm{wt} \%$ ), whereas they reduced with $0.05 \mathrm{wt} \%$ and $0.1 \mathrm{wt} \%$ RGO in our study by up to $51 \%$ and $53 \%$ for the decay time and hysteresis, respectively.

When polymerized, again, RGO can coincide with the polymer not only in the disclinations but also within the DTC, and thus conductivity is efficiently enhanced. When the concentration of RGO is higher than $0.1 \mathrm{wt} \%$, the amount of RGO in the polymer network becomes saturated so that the hysteresis and the decay time become more significant than that in the PSBP. When the RGO concentration is $0.05 \mathrm{wt} \%$ and $0.1 \mathrm{wt} \%$, the molecules in the RGO-polymer network interact efficiently and tightly with the counter-molecules in BPLC, e.g., $\pi-\pi$ interaction between the benzene rings. The $V_{\text {th }}$ and $V_{\text {op }}$, on the other hand, continuously decrease when the RGO concentration increases because the excess RGO outside the polymer still can contribute to the enhancement of conductivity in the system.

FE-SEM was carried out to identify the polymer network [Fig. 5(a)-(c)]. Due to the chiral reactive monomer, the FE-SEM images of the polymer network show an amorphous structure despite the fact that BPI is stabilized. The inset in Fig. 5(c) shows RGO flakes that aggregate when saturated in the RGOpolymer composite. EDX mapping [Fig. 6(a)-(f)] and EDX spectroscopy [Fig. 6(g)-(i)] were carried out to verify the elements in the RGO-polymer network. The carbon element
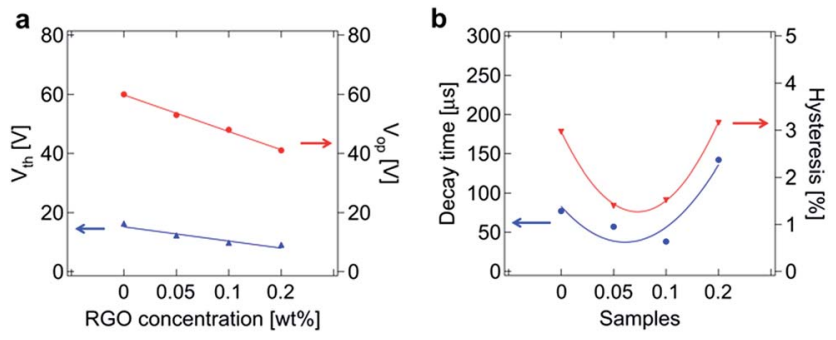

Fig. 4 Measured electro-optic characteristics (at $74{ }^{\circ} \mathrm{C}$ ) of the samples: PSBP, PSBP-RGO-0.05, PSBP-RGO-0.1, PSBP-RGO-0.2. (a) $V_{\text {th }}$ and $V_{\text {op }}$ (b) Decay time and hysteresis.

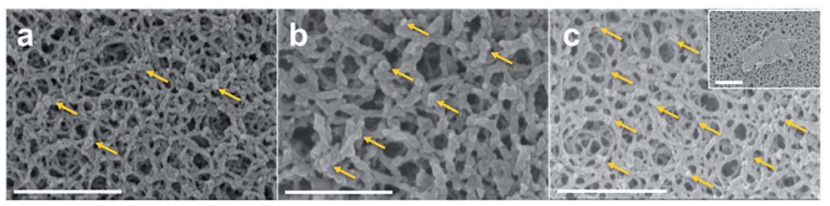

Fig. 5 FE-SEM images of the RGO enriched polymer network with concentrations of (a) 0.05 wt \%, (b) 0.1 wt \% and (c) 0.2 wt\%. Yellow arrows indicate the RGO flakes entangled in the polymer networks. Scale bars are (a, c, and inset in c) $1 \mu \mathrm{m}$, and (b) $500 \mathrm{~nm}$.

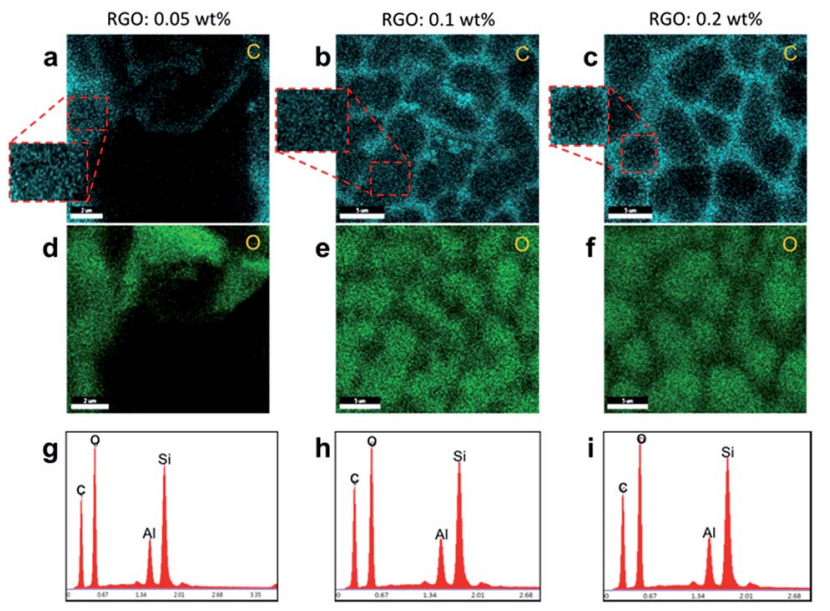

Fig. 6 Characterization of the RGO enriched polymer networks with concentrations of (1st column) $0.05 w t \%$, (2nd column) $0.1 w t \%$, and (3rd column) 0.2 wt\%. EDX maps for $(a-c)$ carbon and $(d-f)$ oxygen with scale bars ( $a$ and d) $2 \mu \mathrm{m}$ and (b, c, e and f) $5 \mu \mathrm{m}$. Insets show that carbon exists within the domains with a relatively lower brightness than that at the boundaries. ( $g-i)$ EDX spectra. (EDX maps do not correspond to the area in the FE-SEM images in Fig. 5(a)-(c), the silicon peak corresponds to the glass substrate in the cell, and the aluminium peak is from the stage that the substrates are loaded on.)

from RGO and oxygen element from the polymer network are indicated by the greenish blue and green colours in Fig. 6(a-c) and (d-f), respectively. The brightest region in the EDX maps corresponds to the area between the different BP domains (as observed in Fig. 2 and 3(b)), and the polymer network of the FESEM images is somewhere inside a domain with a relatively less bright colour. Although the brightness of the colour seems to show a different number of oxygen and RGO flakes exist within the domains and the boundaries, the comparison between the relative amounts in the samples is consistent because the RGO dispersity is the same for each sample.

\section{Experimental}

\section{Synthesis of graphene oxide (GO)}

GO was prepared from natural graphite (Alfa Aesar) using the modified Hummers method., ${ }^{1,2}$

\section{Reduction of GO by hydrazine hydrate}

The prepared GO was reduced by hydrazine hydrate using the following procedure. Firstly, $10 \mathrm{mg}$ of GO was added into $20 \mathrm{ml}$ 
of DI-water $\left(0.5 \mathrm{mg} \mathrm{ml}^{-1}\right)$ and well sonicated to make good dissolution (brown colour). After that, hydrazine hydrate (35 mmol) was added and the solution was maintained at $100^{\circ} \mathrm{C}$ for 16 hours over which the reduced graphene oxide (RGO) gradually precipitated as a black colour. This black precipitate was then filtered by filter paper and washed several times with DIwater and methanol and dried under vacuum to obtain RGO.

\section{Characterization of RGO}

The prepared RGO was characterized via high-resolution transmission electron microscopy (HR-TEM), selected area electron diffraction (SAED), X-ray diffraction (XRD) and X-ray photoelectron spectroscopy (XPS), as shown in Fig. S9(a)-(e), ESI. $\dagger$

\section{Mixtures}

The BPLC mixture consisted of LC (87.8 wt\%; MLC-2053, $\Delta \varepsilon=$ 42.6 and $\Delta n=0.235$ ), a chiral dopant (3.3 wt\%; SRM-17, helical twisting power $(\mathrm{HTP}) \sim 166 \mu \mathrm{m}^{-1}$ ), a photo-polymerizable chiral dopant (1.0 wt\%; SRM-3, HTP $\sim 11 \mu \mathrm{m}^{-1}$ ) [Fig. S9(f) and $(\mathrm{g}), \mathrm{ESI} \dagger]$, reactive diacrylate mesogen $(7.4 \mathrm{wt} \%$; RM-257, $\Delta n$ $=0.179$ ) [Fig. $\mathrm{S} 9(\mathrm{~h}), \mathrm{ESI} \dagger$ ], and a photo-initiator $(0.5 \mathrm{wt} \%$; Irgacure-907). This mixture was then mixed with the desired concentrations of RGO (first dispersed in ethanol and then mixed with the BPLC mixture), and each sample is named in Table 1.

\section{Cells}

After homogeneous mixing, the precursor was heated (at $90{ }^{\circ} \mathrm{C}$ ) until it reached an isotropic phase and then was injected into a unit cell prepared by two glass substrates: one without an electrode and the other with interdigitated indium-tin-oxide (ITO) electrodes (width and space $=4 \mu \mathrm{m}$ ), in which the cell gap was kept by $10 \mu \mathrm{m}$ diameter spacers for the electro-optic characterization. Ultraviolet light $\left(40 \mathrm{~mW} \mathrm{~cm}^{-2}\right.$, wavelength $\lambda$ $=365 \mathrm{~nm}$ ) was irradiated on the samples for polymerstabilization at desired BP temperatures.

\section{Characterization of cells}

The electro-optic properties were measured by placing the cells between crossed polarizers in a way that the longitudinal direction of the electrodes was $45^{\circ}$ to the one of the polarizers and detecting the He-Ne laser light $(\lambda=633 \mathrm{~nm})$ after obtaining the sample layer. By applying voltage to the cell, the in-plane electric field induced birefringence in the PSBP layer governed by the Kerr effect, ${ }^{22,35,36}$ and the transmitted light was detected by a photo-detector connected to an oscilloscope (Agilent 33521A). All the samples employed the same fabrication process and measurement, and the results are summarized in Table 1 (also see the ESI $\dagger$ ). After all the measurements, the cells were submerged in hexane for a day to wash out the BPLCs, and RGO-polymer network was left for observation by fieldemission scanning electron microscopy (FE-SEM) and energydispersive X-ray (EDX) spectroscopy.

\section{Conclusions}

We proposed a reduced graphene oxide (RGO) enriched polymer network that can greatly enhance the electro-optic properties of a polymer-stabilized blue phase (PSBP), such as reduction of the operation voltage, hysteresis and response time in decay. The RGO-polymer composite improves the conductivity of the PSBP and also provides a large number of counterbenzene-rings to liquid crystals for $\pi-\pi$ interaction that highly improves the anchoring energy at the interface between them. We believe that this approach contributes not only to the scientific interest in the effect of RGO-polymer composites on the electro-optic characteristics of PSBP, but also to a breakthrough in the performances of PSBP in device applications.

\section{Acknowledgements}

This research was supported by the Basic Research Laboratory Program (2014R1A4A1008140) through the Ministry of Science, ICT \& Future Planning.

\section{Notes and references}

1 A. K. Geim and K. S. Novoselov, Nat. Mater., 2007, 6, 183-191.

2 J. C. Meyer, A. K. Geim, M. I. Katsnelson, K. S. Novoselov, T. J. Booth and S. Roth, Nature, 2007, 446, 60-63.

3 S. Stankovich, R. D. Piner, X. Chen, N. Wu, S. T. Nguyen and R. S. Ruoff, J. Mater. Chem., 2006, 16, 155-158.

4 S. Stankovich, D. A. Dikin, R. D. Piner, K. A. Kohlhaas, A. Kleinhammes, Y. Jia, Y. Wu, S. T. Nguyen and R. S. Ruoff, Carbon, 2007, 45, 1558-1565.

5 R. Narayan, J. E. Kim, J. Y. Kim, K. E. Lee and S. O. Kim, Adv. Mater., 2016, 28, 3045-3068.

6 J. E. Kim, T. H. Han, S. H. Lee, J. Y. Kim, C. W. Ahn, J. M. Yun and S. O. Kim, Angew. Chem., Int. Ed., 2011, 50, 3043-3047.

7 J. Y. Kim and S. O. Kim, Nat. Mater., 2014, 13, 325-326.

8 K. E. Lee, J. E. Kim, U. N. Maiti, J. Lim, J. O. Hwang, J. Shim, J. J. Oh, T. Yun and S. O. Kim, ACS Nano, 2014, 8, 9073-9080.

9 H. Park, P. R. Brown, V. Bulović and J. Kong, Nano Lett., 2012, 12, 133-140.

10 R. Raccichini, A. Varzi, S. Passerini and B. Scrosati, Nat. Mater., 2015, 14, 271-279.

11 W. Tie, S. S. Bhattacharyya, Y. J. Lim, S. W. Lee, T. H. Lee, Y. H. Lee and S. H. Lee, Opt. Express, 2013, 21, 19867-19879.

12 J.-H. Ahn and B. H. Hong, Nat. Nanotechnol., 2014, 9, 737738.

13 S. Y. Jeong, S. Jeong, S. W. Lee, S. T. Kim, D. Kim, H. J. Jeong, J. T. Han, K.-J. Baeg, S. Yang, M. S. Jeong and G.-W. Lee, Sci. Rep., 2015, 5, 11216.

14 C. J. Kirubaharan, K. Santhakumar, G. G. Kumar, N. Senthilkumar and J.-H. Jang, Int. J. Hydrogen Energy, 2015, 40, 13061-13070.

15 M. V. Kannan and G. G. Kumar, Biosens. Bioelectron., 2016, 77, 1208-1220.

16 C. Zamora-Ledezma, N. Puech, C. Zakri, E. Grelet, S. E. Moulton, G. G. Wallace, S. Gambhir, C. Blanc, 
E. Anglaret and P. Poulin, J. Phys. Chem. Lett., 2012, 3, 24252430.

17 G. G. Kumar, K. J. Babu, K. S. Nahm and Y. J. Hwang, RSC Adv., 2014, 4, 7944.

18 J. Yuan, A. Luna, W. Neri, C. Zakri, T. Schilling, A. Colin and P. Poulin, Nat. Commun., 2015, 6, 8700.

19 S. Ni, H. Li, S. Li, J. Zhu, J. Tan, X. Sun, C. P. Chen, G. He, D. $\mathrm{Wu}$, K.-C. Lee, C.-C. Lo, A. Lien, J. Lu and Y. Su, J. Mater. Chem. C, 2014, 2, 1730.

20 S. Stankovich, D. A. Dikin, G. H. Dommett, K. M. Kohlhaas, E. J. Zimney, E. A. Stach, R. D. Piner, S. T. Nguyen and R. S. Ruoff, Nature, 2006, 442, 282-286.

21 J. W. Doane, A. Golemme, J. L. West, J. B. Whitehead and B.-G. Wu, Mol. Cryst. Liq. Cryst., 1988, 165, 511-532.

22 H. Kikuchi, M. Yokota, Y. Hisakado, H. Yang and T. Kajiyama, Nat. Mater., 2002, 1, 64-68.

23 A. Saupe, Mol. Cryst., 1969, 7, 59-74.

24 S. Meiboom, J. P. Sethna, P. W. Anderson and W. F. Brinkman, Phys. Rev. Lett., 1981, 46, 1216-1219.

25 M. S. Kim and L.-C. Chien, Soft Matter, 2015, 11, 8013-8018.

26 M. S. Kim, Liquid crystalline amorphous blue phase: tangled topological defects, polymer-stabilization, and device application, Electronic Dissertation, Kent State University, OhioLINK Electronic Theses and Dissertations Center, 2015.
27 M. S. Kim, M. Kim, J. H. Jung, K. S. Ha, S. Yoon, E. G. Song, A. K. Srivastava, S.-W. Choi, G.-D. Lee and S. H. Lee, SID Int. Symp. Dig. Tech. Pap., 2009, 40, 1615.

28 M. Kim, M. S. Kim, B. G. Kang, M.-K. Kim, S. Yoon, S. H. Lee, Z. Ge, L. Rao, S. Gauza and S.-T. Wu, J. Phys. D: Appl. Phys., 2009, 42, 235502.

29 K. M. Chen, S. Gauza, H. Xianyu and S. T. Wu, J. Disp. Technol., 2010, 6, 318-322.

30 S. Yoon, M. Kim, M. S. Kim, B. G. Kang, M.-K. Kim, A. K. Srivastava, S. Hee Lee, Z. Ge, L. Rao, S. Gauza and S.-T. Wu, Liq. Cryst., 2010, 37, 201-208.

31 M.-K. Kim, M. S. Kim, B. G. Kang, G. H. Yang, A. Mukherjee, S.-W. Kang, H. Lee, S.-T. Shin and S. H. Lee, Mol. Cryst. Liq. Cryst., 2011, 543, 194.

32 R. Manda, M. S. Kim, E. J. Shin, M.-S. Gong, G. Murali, K. Sandeep, M.-H. Lee, J. H. Lee and S. H. Lee, Liq. Cryst., 2016, 1-10.

33 A. Rani, J. M. Song, M. J. Lee and J. S. Lee, Appl. Phys. Lett., 2012, 101, 233308.

34 R. Islam, A. N. Papathanassiou, R. C. Y. King, J.-F. Brun and F. Roussel, Appl. Phys. Lett., 2015, 107, 53102.

35 M. Sato and A. Yoshizawa, Adv. Mater., 2007, 19, 4145-4148. 36 A. Yoshizawa, $R S C A d v ., 2013,3,25475$. 